\title{
Transcriptional activation of the human S100A2 promoter by wild-type p53
}

\author{
Mingjia Tan ${ }^{\mathrm{a}}$, Claus W. Heizmann ${ }^{\mathrm{b}}$, Kunliang Guan ${ }^{\mathrm{c}}$, Beat W. Schafer ${ }^{\mathrm{b}}$, Yi Sun ${ }^{\mathrm{a}, *}$ \\ ${ }^{a}$ Department of Molecular Biology, Parke-Davis Pharmaceutical Research, Division of Warner-Lambert Company, Ann Arbor, MI 48105, USA \\ ${ }^{\mathrm{b}}$ Department of Pediatrics, Division of Clinical Chemistry and Biochemistry, University of Zurich, Zurich, Switzerland \\ ${ }^{\mathrm{c}}$ Department of Biological Chemistry, University of Michigan, Ann Arbor, MI 48109, USA
}

Received 18 January 1999

\begin{abstract}
S100A2, a calcium binding protein of the EF-hand family, was recently identified to be inducible by etoposide, a p53 activator. A potential $\mathrm{p53}$ binding site was identified in the promoter of the $\mathrm{S100A2}$ gene, which binds to purified $\mathrm{p53}$ as well as $\mathbf{p 5 3}$ in nuclear extract activated by etoposide. Transactivation assays using the promoter driven luciferase reporters revealed that the $\mathrm{S} 100 \mathrm{~A} 2$ promoter was transcriptionally activated by wild-type p53, but not by p53 mutants, in a dose-dependent as well as a p53 binding site-dependent manner. The p53-induced transactivation of the $S 100 A 2$ promoter was enhanced by etoposide and blocked by a dominant negative p53 mutant. Furthermore, endogenous S100A2 mRNA expression is induced by etoposide in $\mathbf{p 5 3}$ positive, but not in $\mathrm{p53}$ negative cells. Thus, p53 appears to positively regulate $\mathrm{S100A2}$ expression.
\end{abstract}

(C) 1999 Federation of European Biochemical Societies.

Key words: p53; S100A2; Calcium binding protein; Transcriptional regulation

\section{Introduction}

p53 is a transcription factor and a tumor suppressor [1]. As a transcription factor, p53 binds to it's consensus binding sequence (two copies of the $10 \mathrm{bp}$ motif $5^{\prime}-\mathrm{PuPuPuC}(\mathrm{A} /$ T)(T/A)GPyPyPy-3', separated by $0-13$ bp) [2] and transactivates a set of target genes. As a tumor suppressor, p53 induces either growth arrest or apoptosis [1]. These biological functions of p53 are mainly mediated through the activation of downstream target genes. For example, p53-induced growth arrest is mainly achieved through activation of p21/Waf-1 [3], whereas p53-induced apoptosis is mediated by or associated with activation of Bax [4], Fas/APO1 [5], KILLER/DR5 [6] and genes involved in generation of reactive oxygen species [7]. Also, potential effects of p53 in tumor metastasis are mediated through regulation of metastasis-related genes $[8,9]$. Thus, identification of genes whose expression is subjected to p53 regulation would lead to a better understanding of p53 functions and its mechanism of action.

In an attempt to identify novel p53 target genes, we treated p53 positive cells with etoposide, a topoisomerase II inhibitor and a p53 activator [8]. Global changes of gene expression were monitored by DNA chip hybridization [10]. Through this approach, we have identified that S100A2, a calcium binding protein $[11,12]$, is inducible by etoposide [10]. We report here the characterization of $\mathrm{p} 53$ regulation of the S100A2 promoter and suggest that the S100A2 is a novel p53 target gene.

\footnotetext{
*Corresponding author. Fax: (1) (734) 622-7158.

E-mail: yi.sun@wl.com
}

\section{Materials and methods}

\subsection{Cell culture and drug treatment}

Two human osteogenic sarcoma cell lines, U2-OS and Saos-2 (ATCC), were grown in McCory or DMEM supplemented with 10\% FCS, respectively. U2-OS cells harbor a wild-type p53 [13], whereas Saos- 2 cells have the p53 gene deleted [14]. For drug treatment, U2-OS and Saos-2 cells were exposed to etoposide $(25 \mu \mathrm{M}$, Sigma) for various periods of time up to $48 \mathrm{~h}$.

\subsection{Gel shift assay}

The assay was performed as described previously [15], using as the probe a 20 bp synthetic oligonucleotide, S100A2.01 (5'-GGGCATGTGTGGGCACGTTC-3'), consisting of the putative p53 binding site identified in the S100A2 promoter and its complementary strand.

\subsection{Luciferase reporter constructions}

The luciferase reporter constructs driven by the S100A2 promoter were made as follows. (a) S100A2 W/p53BS: a 2269 bp DNA fragment of the S100A2 promoter containing the p53 binding site and (b) S100A2 W/O p53BS: a 2060 bp DNA fragment of the S100A2 promoter without $\mathrm{p} 53$ binding site, were generated by PCR amplification from a cloned promoter fragment [16]. The primers used are: PA23Hind (downstream primer): 5'-CTGAAGCTTGGCAGAGACAGACCCAGGAAG-3'; PA25Xho (with p53 site, upstream primer): 5'-CTGCTCGAGTTTGTACAGGACAGAACAGGTAGA-3'; and KA25Xho (without p53 binding site, upstream primer): 5'-AGACTCGAGGGACACGCAGCAGCAGGCC-3'. The PCR fragments were subcloned into pGL-Basic-3 luciferase reporter plasmid (Promega) at the restriction sites of XhoI $\left(5^{\prime}\right)$ and HindIII $\left(3^{\prime}\right)$.

\subsection{DNA transfection and luciferase assay}

Dispersed cells were seeded into 24 well plates at a concentration of $10^{5}$ cells (Saos-2) or $2 \times 10^{5}$ cells (U2-OS) per well 16-24 h prior to transfection. The calcium phosphate method was used to transiently transfect Saos-2 cells [15], whereas the lipofectamine method (BRL) was used for U2-OS transfection according to the manufacturer's instructions. The luciferase reporter constructs described above, along with the empty vector plasmid, were co-transfected with a $\beta$-galactosidase construct in the presence or absence of constructs expressing wild-type or mutant p53 proteins. $38 \mathrm{~h}$ post transfection, cells were lysed and assayed for luciferase/ $\beta$-galactosidase activities [17]. The results are presented as the fold activation over empty reporter after normalizing the $\beta$-galactosidase activity.

\subsection{Northern analysis}

Total RNA was isolated from etoposide-treated cells using RNAzol solution (Tel-Test). $15 \mu \mathrm{g}$ of total RNA was subjected to Northern analysis [18].

\section{Results and discussion}

3.1. Specific binding of wild-type p53 to the p53 binding site identified in the $S 100 A 2$ promoter

Through DNA chip hybridization, we have recently shown that S100A2 is an etoposide-inducible gene [10]. A putative p53 binding site, 5'-GGGCATGTGTGGGCACGGTTC-3' (underlined are two mismatches to the consensus sequence), located at -3811 bp upstream from the translation initiation 


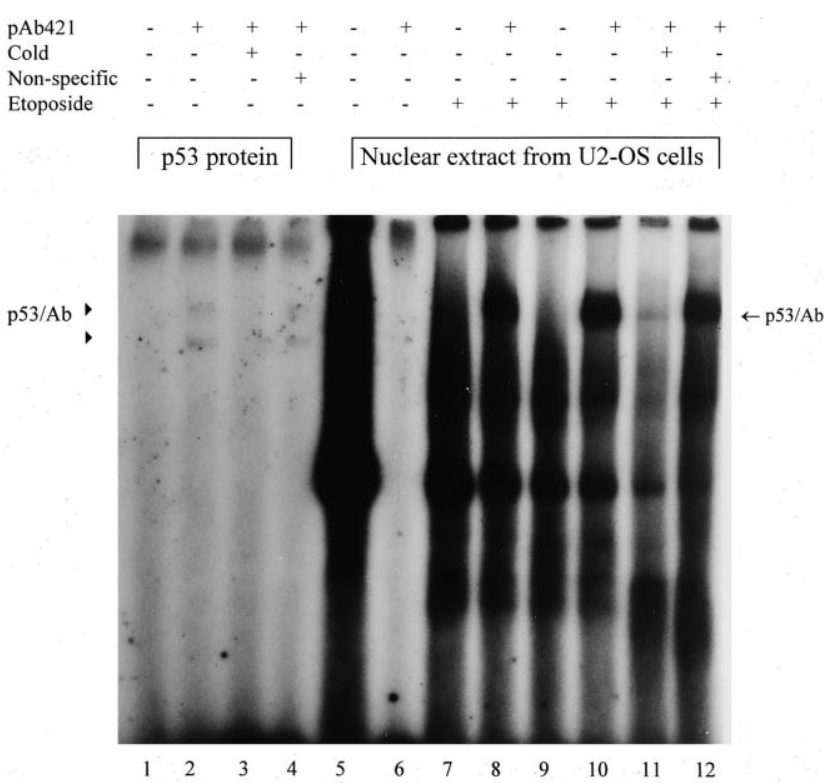

Fig. 1. p53 binds to a putative p53 binding site in the promoter of the S100A2 gene: synthetic oligonucleotides consisting of the p53 binding consensus sequence were labelled with $\left[\gamma^{32} \mathrm{P}\right] \mathrm{ATP}$ using T4 polynucleotide kinase and used in a gel shift assay [15,17]. Lanes 14, partially purified p53 $(3 \mu \mathrm{g})$ with or without pAb421 antibody and lanes 5-12, nuclear extracts $(8.5 \mu \mathrm{g})$ prepared from cells treated with etoposide $(25 \mu \mathrm{M})$ for $0 \mathrm{~h}$ (lanes 5 and 6), $6 \mathrm{~h}$ (lanes 7 and 8) and $24 \mathrm{~h}$ (lanes 9-12). Competition was performed with a 100-fold excess of specific oligonucleotide S100A2.01, or non-specific oligonucleotide mT3SF (5'-GGGGTTGCTTGAAGAGCGTC-3'). The p53$\mathrm{Ab}$ supershifted bands are indicated by the arrows. The band shown on the bottom of the gel is free labelled probe.

site was identified. To examine whether p53 is able to bind to this putative binding site, we used both purified p53 protein [17] and nuclear extract from p53 positive U2-OS cells treated with etoposide $(25 \mu \mathrm{M})$ for 0,6 or $24 \mathrm{~h}$. As shown in Fig. 1, a $20 \mathrm{bp}$ oligonucleotide, consisting of the S100A2 p53 binding site, binds to purified p53 only in the presence of p53 antibody, pAb421, that is known to enhance and stabilize p53 DNA binding [19] (compare lanes 1 and 2). Two binding bands are revealed (as indicated by arrows), possibly reflecting different conformations [17]. The binding is sequence-specific since it can be blocked by a specific (lane 3 ) but not by a nonspecific cold oligonucleotide (lane 4). Endogenous p53 in unstimulated U2-OS is found not to be capable of binding to the oligonucleotide due to the low protein level (lanes 5 and 6). Upon activation by etoposide for 6 or $24 \mathrm{~h}$, however, a strong p53 binding is detected in the presence of antibody (lanes 8 and 10). Again, the binding is sequence-specific and can be blocked by addition of an excess of unlabelled specific oligonucleotide (lane 11), but not by a non-specific control oligonucleotide (lane 12). The result indicates that $\mathrm{p} 53$ can bind to the putative p53 binding site in the S100A2 promoter.

\subsection{Transactivation of the $S 100 A 2$ promoter by p53 is both p53 dose- and p53 binding site-dependent}

We first examined whether p53 transactivates the S100A2 promoter and whether activation is p53 dose-dependent. We generated a luciferase reporter construct driven by a $2269 \mathrm{bp}$ promoter fragment containing the p53 binding sites (S100A2 $\mathrm{W} / \mathrm{p} 53 \mathrm{BS}$ ) and used it in a transactivation/luciferase assay. The result shows that $\mathrm{p} 53$ does transactivate the S100A2 pro- p53-dependent activation of the S100A2 promoter

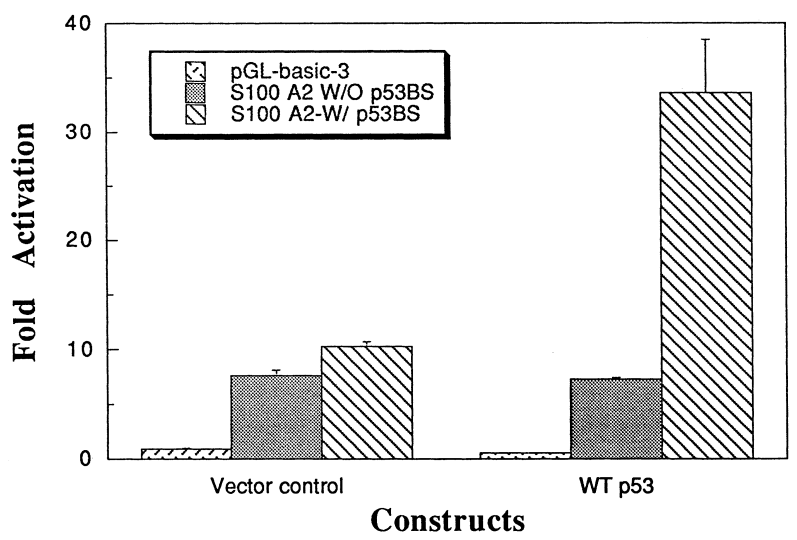

Fig. 2. p53 binding site-dependent activation of the S100A2 promoter. Two luciferase reporter constructs driven by the S100A2 promoter with (S100A2 W/p53BS) or without (S100A2 W/O p53BS) the p53 binding site were transiently co-transfected with or without p53 expressing plasmid, respectively, into human Saos-2 cells. The results are presented as fold activation \pm S.D. derived from three independent transfections, each run in duplicate, after normalization with the $\beta$-galactosidase activity for the transfection efficiency.

moter and transactivation is p53 dose-dependent (data not shown). We next examined whether p53-induced transactivation of the S100A2 promoter is p53 binding site-dependent. We compared luciferase activity in the constructs driven by the S100A2 promoter sequence with (S100A2 W/p53BS) or without (S100A2 W/O p53BS) p53 binding site in Saos-2 cells after transiently co-transfected with a p53 expressing plasmid or an empty vector as the control. As shown in Fig. 2, in the absence of p53 (vector control), both constructs produce a comparable promoter activity, giving rise to a $8-10$-fold higher luciferase activity than that of the empty vector. Most likely, this activity reflects the level of endogenous S100A2 expression generated by the enhancer element identified earlier [16]. In the presence of p53, the construct without p53 binding site gives rise to a similar level of luciferase activity. However,

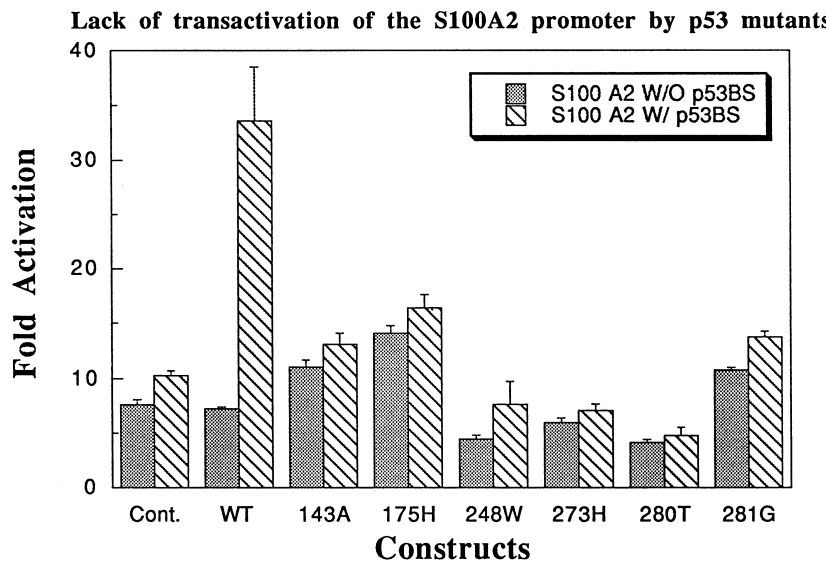

Fig. 3. Tumor-derived p53 mutants do not transactivate the S100A2 promoter. The constructs S100A W/p53BS and S100A2 W/O p53BS were transiently co-transfected, respectively, with plasmids expressing p53 mutants into human Saos-2 cells. The results are presented as fold activation \pm S.D. derived from three independent transfections and assays, each run in duplicate, after normalization with the $\beta$-galactosidase activity for the transfection efficiency. 
Activation of the $\mathrm{S} 100$ A2 promoter by etoposide
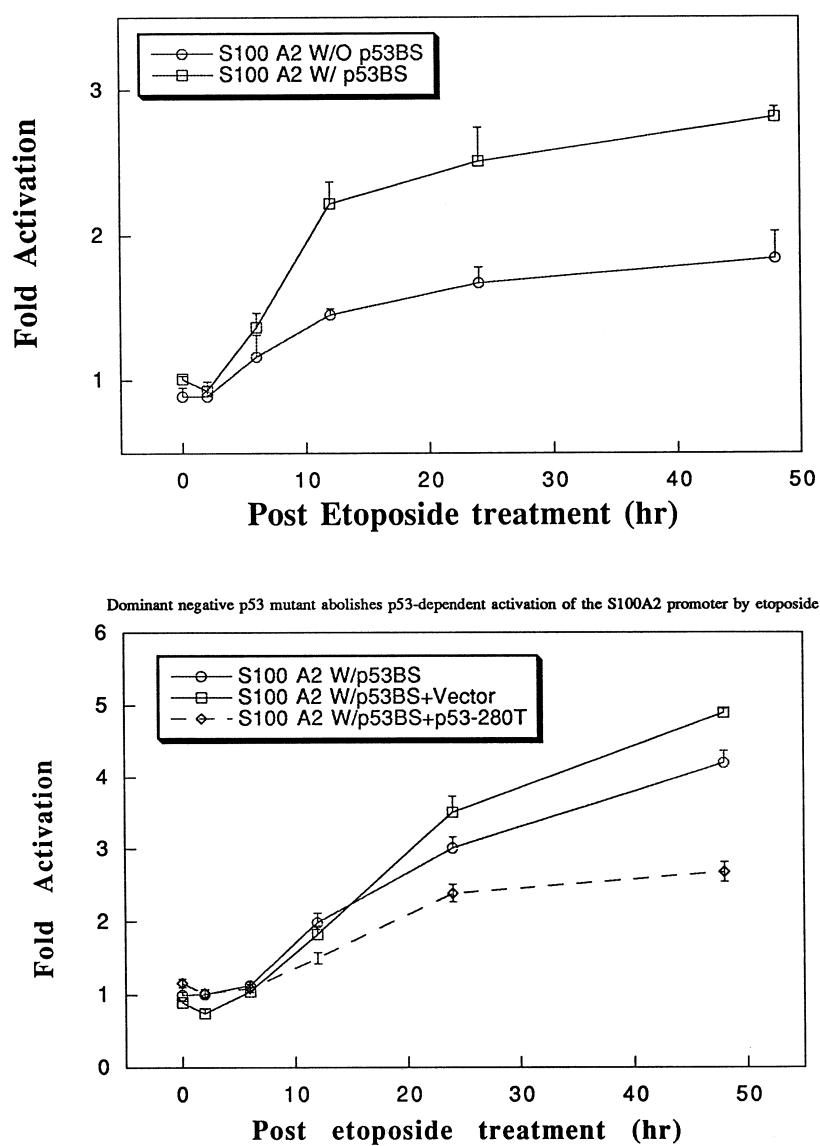

Fig. 4. Induction of p53-dependent transactivation of the S100A2 promoter by etoposide. Subconfluent U2-OS cells were co-transfected with S100A2 W/p53BS or W/O p53BS luciferase reporters (top) and p53 mutant expressing construct (p53-280T) or the vector control (bottom). Cells were treated with etoposide $(25 \mu \mathrm{M}) 24 \mathrm{~h}$ post transfection for $0,2,6,12,24$ or $48 \mathrm{~h}$, respectively, followed by luciferase activity measurements. Three independent transfections, each run in duplicate, were performed and results are presented as fold activation \pm S.D. after normalization with the $\beta$-galactosidase activity for the transfection efficiency. To calculate the fold activation, the luciferase activity from the S100A2 W/O p53BS construct (top) or from the S100A2 W/p53BS construct (bottom) after $0 \mathrm{~h}$ etoposide treatment was arbitrarily set as 1 .

the p53 binding site-containing construct induces a 35 -fold activation of luciferase activity. Hence, p53 can stimulate promoter activity 3.5 -fold over background levels. These results indicate that transactivation of the S100A2 promoter by $\mathrm{p} 53$ is p53 binding site-dependent.

\subsection{Lack of transactivation of the $\$ 100 A 2$ promoter by p53 mutants}

We further examined whether the S100A2 promoter activity could also be stimulated by p53 mutants. Plasmid DNAs expressing p53 mutant proteins were individually co-transfected with luciferase reporters into Saos- 2 cells. Mutants used are p53-143A, p53-175H, p53-248W, p53-273H, p53-281G, five of the most common p53 mutants in human cancers [20] and p53-280T, a dominant negative p53 mutant found in nasopharyngeal carcinomas [21-23]. As shown in Fig. 3, no significant induction of luciferase activity is observed by any of the p53 mutants. These results indicate that all p53 mutants have lost the ability to transactivate the S100A2 promoter.
3.4. Induction of p53-dependent transactivation by etoposide

Etoposide has been previously shown to activate p53 in U2OS cells [8]. We therefore examined whether etoposide would induce p53-dependent transactivation of the S100A2 promoter. As shown in Fig. 4 (top panel), transactivation of the luciferase reporter driven by the p53 site-containing promoter is induced by etoposide with a 3-fold induction achieved at $48 \mathrm{~h}$. A slight induction of the luciferase activity was also seen in the $\mathrm{p} 53$ site-deleted promoter that appears to be $\mathrm{p} 53$-independent. To confirm that etoposide-induced transactivation is largely p53-dependent, we transfected p53-280T, a known dominant negative p53 mutant [21-23] into U2-OS cells, followed by etoposide treatment. As a control, the empty vector was used. As shown in Fig. 4 (bottom panel), etoposide-induced activation is abolished by p53-280T $(\mathrm{S} 100 \mathrm{~A} 2 \mathrm{~W} / \mathrm{p} 53 \mathrm{BS}+\mathrm{p} 53-280 \mathrm{~T})$ to a level comparable with that of S100A2 W/O p53BS (Fig. 4, top panel), but it is not inhibited by the vector control (S100A2 W/p53BS+Vector). These results demonstrate that transactivation of the S100A2 promoter by etoposide is largely p53-dependent.

\subsection{Induction of $S 100 A 2 \mathrm{mRNA}$ expression by etoposide in p53 positive, but not in p53 negative cells}

To examine whether endogenous S100A2 is subjected to p53 regulation, we treated p53 positive U2-OS cells and p53 negative Saos-2 cells with etoposide. As shown in Fig. 5, there is a basal level of S100A2 mRNA in U2-OS cells, which is induced by a 3-fold after exposure to etoposide for $24 \mathrm{~h}$. In Saos-2 cells, S100A2 mRNA is not detectable at the basal level and no induction is observed by etoposide. The result indicates a p53 status-dependent regulation of S100A2 expression.

S100A2 is an EF-hand calcium binding protein that has been associated with the development of malignancies through its down-regulation during tumor progression [12,24]. This down-regulation has previously been suggested to depend upon the DNA methylation status in the promoter region $[16,25]$. On the other hand, as shown in this report, the $\mathrm{S} 100 \mathrm{~A} 2$ promoter activity can also be influenced by the $\mathrm{p} 53$ status of tumor cells. Hence, the loss of S100A2 expression in tumor cells is mediated by at least two independent mechanisms. The finding that the S100A2 promoter is subjected to positive regulation by wild-type p53, but not by any p53 mutants appears to be significant since it links the p53 signalling

\section{U2-OS SAOS-2}
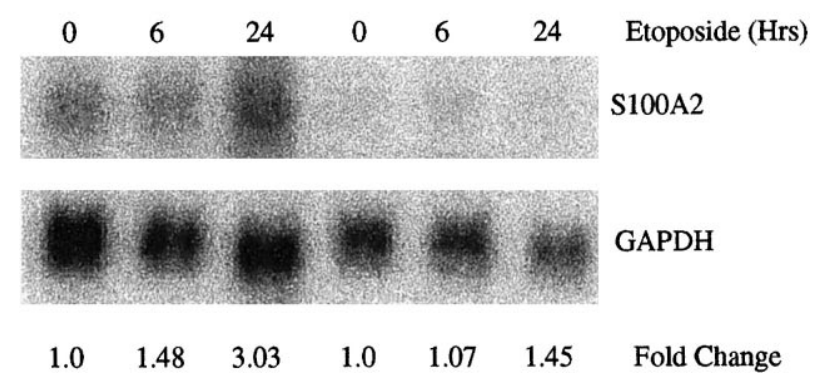

Fig. 5. Induction of endogenous S100A2 mRNA expression by etoposide in p53 positive cells. Total RNA was isolated from p53 positive U2-OS and p53 negative Saos-2 cells treated with etoposide (25 $\mu \mathrm{M})$ for 0,6 or $24 \mathrm{~h}$ and subjected $(15 \mu \mathrm{g})$ to Northern analysis. The fold change after normalization with GAPDH is listed on the bottom of the figure. 
pathway to the calcium signalling pathway, both of which are involved in apoptosis [26,27]. It could imply that S100 family members mediate p53 apoptosis signals. Indeed, S100B, a second S100 family member was recently found to functionally interact with p53 in the regulation of p53-dependent cell growth arrest and apoptosis [28]. Interestingly, the expression of S100B was also increased in cells expressing wild-type p53 in response to UV irradiation [28,29]. Since S100A2 is a nuclear protein [11], it might also be possible that it can functionally interact with p53 as shown for S100B [28,29]. Thus, our finding that $\mathrm{S} 100 \mathrm{~A} 2$ is a novel p53 target gene suggests possible concerted functions of S100A2 and p53 in mediating the tumor suppressor role of $\mathrm{p} 53$.

Acknowledgements: This work was partly supported by the Swiss National Science Foundation (31-50510.97 to BWS). We thank P. Murmann for generating the S100A2 promoter constructs.

\section{References}

[1] Levine, A.J. (1997) Cell 88, 323-331.

[2] el Deiry, W.S., Kern, S.E., Pietenpol, J.A., Kinzler, K.W. and Vogelstein, B. (1992) Nat. Genet. 1, 45-49.

[3] el Deiry, W.S. et al. (1993) Cell 75, 817-825.

[4] Knudson, C.M., Tung, K.S., Tourtellotte, W.G., Brown, G.A. and Korsmeyer, S.J. (1995) Science 270, 96-99.

[5] Fuchs, E.J., McKenna, K.A. and Bedi, A. (1997) Cancer Res. 57, $2550-2554$.

[6] Wu, G.S. et al. (1997) Nat. Genet. 17, 141-143.

[7] Polyak, K., Xia, Y., Zweier, J.L., Kinzler, K.W. and Vogelstein, B. (1997) Nature 389, 300-305.

[8] Bian, J. and Sun, Y. (1997) Mol. Cell Biol. 17, 6330-6338.

[9] Sun, Y., Wicha, M. and Leopold, W.R. (1999) Mol. Carcinog., (in press).
[10] Wang, Y., Rea, T., Bian, J., Gray, S. and Sun, Y. (1999) FEBS Lett. 445, 269-273.

[11] Franz, C., Durussel, I., Cox, J.A., Schafer, B.W. and Heizmann, C.W. (1998) J. Biol. Chem. 273, 18826-18834.

[12] Schafer, B.W. and Heizmann, C.W. (1996) Trends Biochem. Sci. 21, 134-140.

[13] Kastan, M.B. et al. (1992) Cell 71, 587-597.

[14] Masuda, H., Miller, C., Koeffler, H.P., Battifora, H. and Cline, M.J. (1987) Proc. Natl. Acad. Sci. USA 84, 7716-7719.

[15] Sun, Y., Bian, J., Wang, Y. and Jacobs, C. (1997) Oncogene 14, 385-393.

[16] Wicki, R., Franz, C., Scholl, F.A., Heizmann, C.W. and Schafer, B.W. (1997) Cell Calcium 22, 243-254.

[17] Bian, J., Jacobs, C., Wang, Y. and Sun, Y. (1996) Carcinogenesis $17,2559-2562$.

[18] Sun, Y., Pommier, Y. and Colburn, N.H. (1992) Cancer Res. 52, 1907-1915.

[19] Hupp, T.R., Meek, D.W., Midgley, C.A. and Lane, D.P. (1992) Cell 71, 875-886.

[20] Hollstein, M., Sidransky, D., Vogelstein, B. and Harris, C.C. (1991) Science 253, 49-53.

[21] Sun, Y., Nakamura, K., Wendel, E. and Colburn, N. (1993) Proc. Natl. Acad. Sci. USA 90, 2827-2831.

[22] Sun, Y., Dong, Z., Nakamura, K. and Colburn, N.H. (1993) FASEB J. 7, 944-950.

[23] Wallingford, J.B., Seufert, D.W., Virta, V.C. and Vize, P.D. (1997) Curr. Biol. 7, 747-757.

[24] Lee, S.W., Tomasetto, C., Swisshelm, K., Keyomarsi, K. and Sager, R. (1992) Proc. Natl. Acad. Sci. USA 89, 2504-2508.

[25] Lee, S.W., Tomasetto, C. and Sager, R. (1991) Proc. Natl. Acad. Sci. USA 88, 2825-2829.

[26] Ko, L.J. and Prives, C. (1996) Genes Dev. 10, 1054-1072.

[27] Berridge, M.J., Bootman, M.D. and Lipp, P. (1998) Nature 395, 645-648.

[28] Scotto, C., Deloulme, J.C., Rousseau, D., Chambaz, E. and Baudier, J. (1998) Mol. Cell Biol. 18, 4272-4281.

[29] Rustandi, R.R., Drohat, A.C., Baldisseri, D.M., Wilder, P.T. and Weber, D.J. (1998) Biochemistry 37, 1951-1960. 